\title{
Three-Dimensional Structure of Non-Equilibrium Homogeneous Condensation Flow in a Supersonic Rectangular Nozzle
}

\author{
Shibanul Haque ${ }^{1}$, Shigeru Matsuo², Heuy-Dong Kim³ \\ ${ }^{1}$ Graduate School of Science \& Engineering, Saga University, Saga, Japan \\ ${ }^{2}$ Department of Science and Engineering, Saga University, Saga, Japan \\ ${ }^{3}$ School of Mechanical Engineering, Andong National University, Andong, Korea \\ Email:20706013@edu.cc.saga-u.ac.jp
}

How to cite this paper: Haque, S., Matsuo, S. and Kim, H.-D. (2021) Three-Dimensional Structure of Non-Equilibrium Homogeneous Condensation Flow in a Supersonic Rectangular Nozzle. Open Journal of Fluid Dynamics, 11, 55-66.

https://doi.org/10.4236/ojfd.2021.112004

Received: February 9, 2021

Accepted: April 5, 2021

Published: April 8, 2021

Copyright $\odot 2021$ by author(s) and Scientific Research Publishing Inc. This work is licensed under the Creative Commons Attribution International License (CC BY 4.0).

http://creativecommons.org/licenses/by/4.0/

\begin{abstract}
Numerical simulations have been carried out for a supersonic three-dimensional rectangular arc nozzle, where a secondary flow toward the center of the curvature occurs due to the shape of the nozzle. It is known that secondary flow causes longitudinal vortices to form near the wall of the nozzle corner, making the nozzle outlet flow unstable and induces loss of transport energy. When the working fluid is a condensable gas with relatively large latent heat such as moist air or steam, rapid accelerated expansion in the nozzle causes non-equilibrium condensation due to supersaturation. After the release of latent heat during phase transition, nozzle flow continues expanding at an equilibrium saturation condition. In the absence of foreign particles, e.g. ions or dust particles, condensation nuclei are formed in the gas itself causing non-equilibrium homogeneous condensation. Supersonic nozzle flow properties vary considerably due to the occurrence of condensation phenomenon. The objective of this study is to investigate the effect of non-equilibrium homogeneous condensation on the longitudinal vortices which form in the range close to the corner of rectangular arc nozzle numerically.
\end{abstract}

\section{Keywords}

Compressible Flow, Non-Equilibrium Condensation, Supersonic Nozzle, Vortex, Simulation

\section{Introduction}

The instability of a boundary layer over the concave surface results from the imbalance between the centrifugal force and the wall-normal pressure gradient in 
case of supersonic rectangular nozzle flow [1]. As a result of interaction between the two adjacent boundary layers, driven by the lateral pressure gradient; stream-oriented, counter-rotating longitudinal vortices are created and can be significantly intensified downstream of the flow causing flow transition. This transition of the flow from laminar to turbulent prevents the quiet nature of the rectangular wind tunnel [2]. Besides, successful design of the turbomachinery requires an understanding of the secondary flow. This flow is generated when a developed flow passes through a bend, or when a sheared flow passes over an aerofoil, or when a boundary layer faces an obstruction normal to the flow direction. It is well known that in a turbomachine there are three major losses: profile losses, end wall secondary flow losses, and tip clearance losses. These losses are accountable for almost 30 - 50 percent of the total pressure loss in a blade row passage [3]. Prediction of the cascade vorticity by the classical theory of secondary flow by Howthorne [4] showed the presence of end wall vortices.

There has not been much study about the interference between non-equilibrium homogeneous condensation and the corner flow vortices. In general, air as a working fluid contains humidity which induces non-equilibrium homogeneous condensation due to adiabatic expansion. Therefore, it is important to understand the effect of non-equilibrium homogeneous condensation on the corner flow vortices in order to delay the laminar-turbulent flow transition and to reduce the total pressure loss.

In case of supersonic rectangular nozzle flow, high-speed expansion of moist air causes the formation of condensate particles [5]. During condensation, change of phases occurs from water vapor into liquid or liquid into solid phase. This phenomenon causes release of latent heat due to the phase change resulting in an increase of specific volume which causes reduction in gas mass flow rate. Molecular weight, gas constant, specific heat of the flow also change with condensation process [6]. In the absence of foreign nuclei, homogeneous condensation takes place through the formation of water nuclei from its vapor. The purpose behind the development of the homogeneous nucleation theory was to predict condensation and to apply this theory to various devices such as wind tunnel and steam turbine nozzle etc. [7]-[13]. Condensation and nucleation phenomena have great importance in compressible fluid dynamics because it contains two-phase flow and multi-component system. Two-phase flow demands significant attention due to the thermo-fluid dynamics that take place during the change of phase. The characteristics of the supersonic nozzle flow properties vary greatly due to the existence of condensate particles and the flight simulation in the wind tunnel would not experience the isentropic fluid flow due to the presence of condensate particles [14]. Besides, airflow experiences pressure and temperature drop due to the rapid expansion of the flow in a supersonic rectangular nozzle. This rapid expansion causes supersaturation with respect to the equilibrium state due to the absence of condensate deposit surface.

This research aims to investigate the effect of non-equilibrium homogeneous condensation on the corner flow of a three-dimensional supersonic rectangular 
arc nozzle numerically. Density distributions along with the flow vorticity near the nozzle corner have been analyzed to know the influence of non-equilibrium homogeneous condensation on the flow vortices in cases of dry air and moist air.

\section{Numerical Analysis}

In this research, numerical calculations were performed using the unsteady three-dimensional compressible Navier-Stokes equations in combination with the equations of continuity, energy, turbulent kinetic energy, specific dissipation rate, conservation of mass of the liquid phase, and conservation of the number density of droplets with homogeneous nucleation. Discretization was performed spatially by the cell-centered type finite volume method. Furthermore, a third-order accurate MUSCL TVD (Total Variation Diminishing) schemes based on Roe's approximate Riemann solver [15] were applied to the inviscid fluxes and the viscous fluxes were evaluated by the second-order accurate central difference method. Generation terms associated with turbulence and condensation were evaluated by the first-order accurate central difference method. Unfactored implicit equations derived with no approximate factorization were solved by a point Gauss-Seidel relaxation method [16].

$$
\frac{\partial \boldsymbol{Q}}{\partial t}+\frac{\partial \boldsymbol{E}}{\partial x}+\frac{\partial \boldsymbol{F}}{\partial y}+\frac{\partial \boldsymbol{G}}{\partial z}=\frac{\partial \boldsymbol{E}_{\mathrm{v}}}{\partial x}+\frac{\partial \boldsymbol{F}_{\mathrm{v}}}{\partial y}+\frac{\partial \boldsymbol{G}_{\mathrm{v}}}{\partial z}+\boldsymbol{I}+\boldsymbol{S}
$$

where $Q$ is the conservative vector; $E, F$ and $G$ are inviscid flux vectors; $E_{\mathrm{v}}, F_{\mathrm{v}}, G_{\mathrm{v}}$ are viscosity flux vectors. $I$ and $S$ are the source terms corresponding to turbulence and condensation, respectively. The governing equation systems that are non-dimensionalized with reference values at the inlet conditions upstream of the nozzle are mapped from the physical plane into a computational plane of a general transform.

$$
\boldsymbol{Q}=\left[\begin{array}{c}
\rho_{\mathrm{m}} \\
\rho_{\mathrm{m}} u \\
\rho_{\mathrm{m}} v \\
\rho_{\mathrm{m}} w \\
E_{\mathrm{s}} \\
\rho_{\mathrm{m}} k_{\mathrm{t}} \\
\rho_{\mathrm{m}} k_{\mathrm{l}} \\
\rho_{\mathrm{m}} \omega \\
\rho_{\mathrm{m}} g \\
\rho_{\mathrm{m}} n
\end{array}\right], \quad \boldsymbol{E}=\left[\begin{array}{c}
\rho_{\mathrm{m}} u \\
\rho_{\mathrm{m}} u^{2}+p \\
\rho_{\mathrm{m}} u v \\
\rho_{\mathrm{m}} u w \\
\left(E_{\mathrm{s}}+p\right) u \\
\rho_{\mathrm{m}} k_{\mathrm{t}} u \\
\rho_{\mathrm{m}} k_{\mathrm{l}} u \\
\rho_{\mathrm{m}} \omega u \\
\rho_{\mathrm{m}} g u \\
\rho_{\mathrm{m}} n u
\end{array}\right], \boldsymbol{F}=\left[\begin{array}{c}
\rho_{\mathrm{m}} v \\
\rho_{\mathrm{m}} u v \\
\rho_{\mathrm{m}} v^{2}+p \\
\rho_{\mathrm{m}} v w \\
\left(E_{\mathrm{s}}+p\right) v \\
\rho_{\mathrm{m}} k_{\mathrm{t}} v \\
\rho_{\mathrm{m}} k_{\mathrm{l}} v \\
\rho_{\mathrm{m}} \omega v \\
\rho_{\mathrm{m}} v g \\
\rho_{\mathrm{m}} v n
\end{array}\right], \boldsymbol{G}=\left[\begin{array}{c}
\rho_{\mathrm{m}} w \\
\rho_{\mathrm{m}} u w \\
\rho_{\mathrm{m}} v w \\
\rho_{\mathrm{m}} w^{2}+p \\
\left(E_{\mathrm{s}}+p\right) w \\
\rho_{\mathrm{m}} k_{\mathrm{t}} w \\
\rho_{\mathrm{m}} k_{\mathrm{l}} w \\
\rho_{\mathrm{m}} \omega w \\
\rho_{\mathrm{m}} w g \\
\rho_{\mathrm{m}} w n
\end{array}\right]
$$

Numerical modelling of non-equilibrium homogeneous condensation phenomenon consists of two equations for condensate mass fraction $g$ and the number of droplets per unit mass $n$ [17].

$$
\frac{\partial\left(\rho_{\mathrm{m}} g\right)}{\partial t}+\frac{\partial\left(\rho_{\mathrm{m}} g u\right)}{\partial x}+\frac{\partial\left(\rho_{\mathrm{m}} g v\right)}{\partial y}+\frac{\partial\left(\rho_{\mathrm{m}} g w\right)}{\partial \mathrm{z}}=\frac{4 \pi}{3} \rho_{\mathrm{l}}\left(r_{\mathrm{c}}^{3} I_{\mathrm{F}}+3 \rho_{\mathrm{m}} n r^{2} \frac{\mathrm{d} r}{\mathrm{~d} t}\right)
$$




$$
\frac{\partial\left(\rho_{\mathrm{m}} n\right)}{\partial t}+\frac{\partial\left(\rho_{\mathrm{m}} n u\right)}{\partial t}+\frac{\partial\left(\rho_{\mathrm{m}} n v\right)}{\partial t}+\frac{\partial\left(\rho_{\mathrm{m}} n w\right)}{\partial t}=I_{\mathrm{F}}
$$

Reference [18] provided the equations of latent heat, critical radius of the nuclei, droplet average radius, radius growth rate, density of liquid phase, and surface tension. While Frenkel's equation [19] was used to calculate nucleation rate. Numerical calculations of the flow over a flat plate using $k_{\mathrm{t}}-k_{1}-\omega$ turbulence model [20] have been performed to set the initial values of turbulent kinetic energy $k_{t 0}$, laminar kinetic energy $k_{10}$ and specific dissipation rate $\omega_{0}$ in case of rectangular arc nozzle flow. The $y^{+}-u^{+}$distribution of the flow in case of flat plate was consistent with the experimental value [21] which validated the simulation.

\section{Calculation Domain and Conditions}

Figure 1 shows the calculation domain and boundary conditions of the supersonic rectangular arc nozzle flow. The height at entrance and exit of the nozzle is $60 \mathrm{~mm}$, while the width is $19 \mathrm{~mm}$. The height of the throat $H$ (characteristic length) is $23.8 \mathrm{~mm}$ and the nozzle arc radius of curvature is $400 \mathrm{~mm}$. Total length of the nozzle is $550 \mathrm{~mm}$ while the supersonic portion of the nozzle is 310 mm long. This simulation was conducted by considering only half of the nozzle span direction to reduce the calculation time. The solid wall boundary condition was no-slip, while slip boundary condition was applied at the beginning of the nozzle span direction.

Initial calculation conditions were fixed at the nozzle inlet while the back pressure was not fixed at the nozzle outlet. Initial stagnation pressure $p_{0}$, temperature $T_{0}$, and initial degree of relative humidity $\phi_{0}$ were set at $102 \mathrm{kPa}, 287$ $\mathrm{K}$ and $60 \%$, respectively, in case of moist air. In addition, initial stagnation pressure $p_{0}$, temperature $T_{0}$, and initial relative humidity $\phi_{0}$ were set at $102 \mathrm{kPa}$,

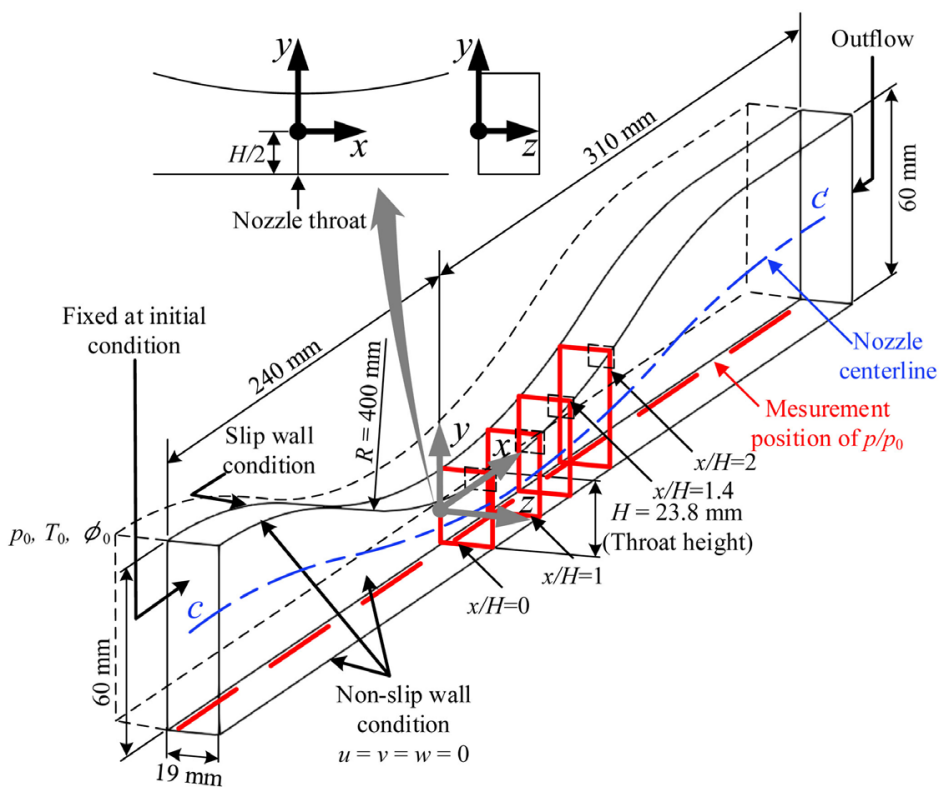

Figure 1. Nozzle geometry and boundary conditions. 
$287 \mathrm{~K}$ and $0 \%$, respectively, in case of dry air. In both cases, initial turbulent kinetic energy $k_{\mathrm{t} 0}$, laminar kinetic energy $k_{10}$ and specific dissipation rate $\omega_{0}$ were set at $0.124490 \mathrm{~J} / \mathrm{kg}, 0 \mathrm{~J} / \mathrm{kg}$, and $364.955 \mathrm{1} / \mathrm{s}$, respectively.

\section{Results and Discussion}

Simulations of the rectangular supersonic arc nozzle flow in cases of dry air and moist air have been carried out until the residual error has reached the order of $10^{-6}$. In Figure 1, a dashed red line close to the bottom of the nozzle center indicates the measurement position of the static pressure distribution. In order to know the effect of non-equilibrium homogeneous condensation on the nozzle corner flow, four cross-sectional views were examined at $x / H=0,1,1.4$, and 2 , respectively. Here, $x / H=0$ indicates nozzle throat, while non-equilibrium homogeneous condensation zone is located in the range from $x / H=1$ to $x / H=1.4$, and $x / H=2$ represents a position after the non-equilibrium condensation zone.

As shown in Figure 2, static pressure distributions obtained by the simulations in cases of dry and moist air are almost similar to the experimental datum [22], which eventually validates the calculation. The onset of non-equilibrium homogeneous condensation in case of moist air causes an increase in static pressure distribution downstream of the nozzle throat. In addition, carefully identified four positions are shown to investigate the effect of non-equilibrium homogeneous condensation on the longitudinal vortices at the nozzle corner flow.

Distributions of condensate mass fraction $g$, nucleation rate $I_{\mathrm{F}}$, and static pressure $p / p_{0}$ are illustrated for the non-equilibrium homogeneous condensation phenomenon of moist air in Figure 3. As the condensable moist air expands rapidly in this supersonic nozzle, the release of latent heat with the onset of non-equilibrium homogeneous condensation causes an increase in static pressure due to the deviation from the isentropic flow around $x / H=1$. The nucleation rate reaches its maximum value at the onset of non-equilibrium homogeneous condensation and the condensate mass fraction $g$ begins to increase.

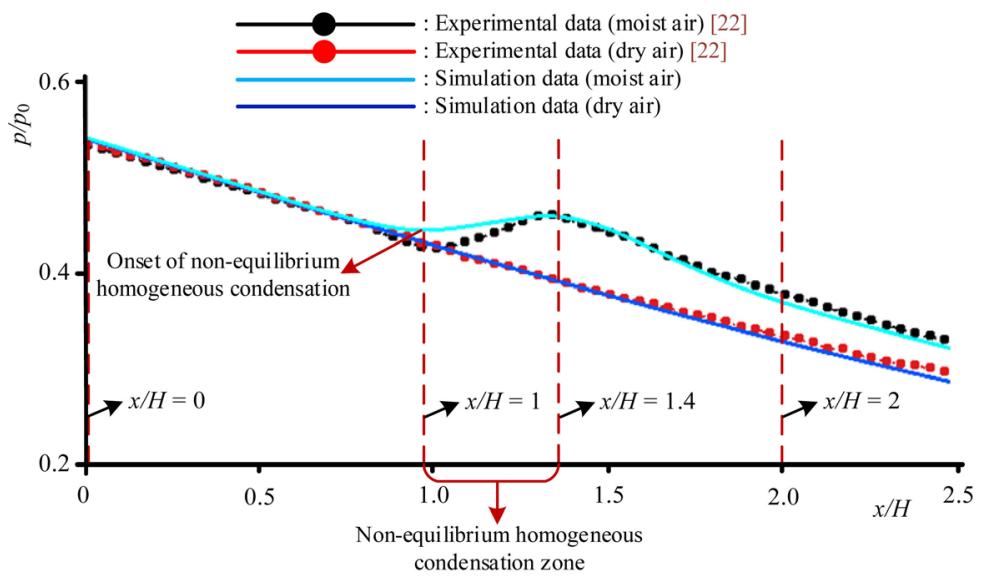

Figure 2. Stream-wise distributions of $p / p_{0}$ in case of dry air and moist air on the center line of bottom wall. 


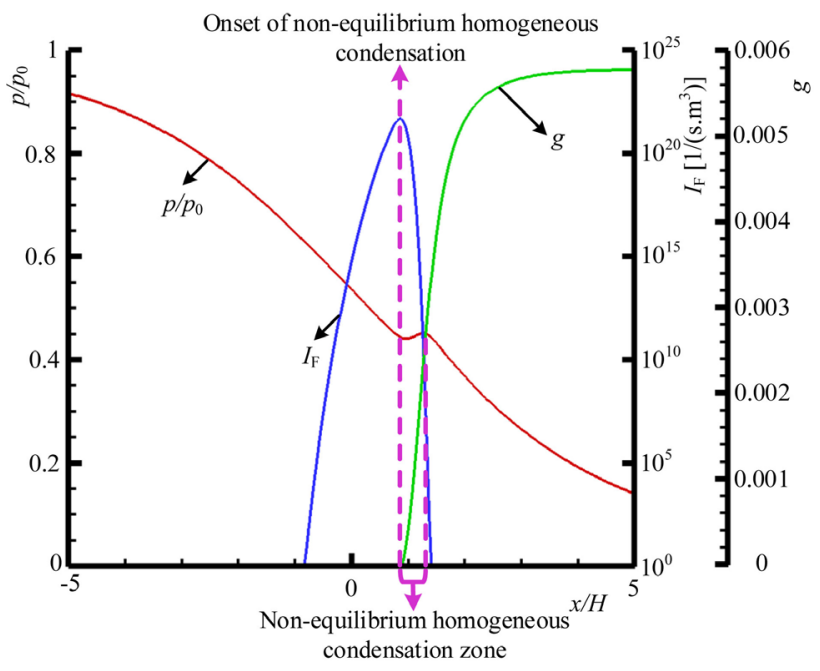

Figure 3. Distributions of condensate mass fraction $g$, nucleation rate $I_{\mathrm{F}}$, and static pressure $p / p_{0}$ at the measurement line $\left(\mathrm{c}-\mathrm{c}^{\prime}\right)$.

Besides, the end of non-equilibrium condensation zone at $x / H=1.4$ is indicated by the maximum point of the static pressure rise and after this point the flow again becomes isentropic.

Figure 4 illustrates the onset of non-equilibrium homogeneous condensation with contour map of condensate mass fraction $g$. It can be seen that the non-equilibrium homogeneous condensation forms in a gentle curved surface from the upstream upper wall side to the downstream lower wall of the nozzle. Besides, it is found that the surface of condensation onset has a complicated structure due to an interaction between the boundary layer and the longitudinal vortex at the nozzle corner flow which is shown in the $y$ - $z$ plane of Figure 4(b). The $y-z$ plane contains the front view of the condensate mass fraction contour map in the flow direction.

In case of dry air, Figure 5 shows contour maps of vorticity $(\omega)$ near the corner of the nozzle upper wall at four positions $(x / H=0$ (nozzle throat), 1.0, 1.4, and 2.0). Density distributions of each cross-sectional plane are shown in the $y$-axis direction at $z / H=0.40,0.714,0.742,0.762$, and 0.779 , respectively. Horizontal dotted lines illustrate the position of $\delta_{\text {dry }}$, where the absolute value of density gradient $(d \rho / d y)$ is minimum and $\delta_{\text {drymax }}$ is the maximum thickness of $\delta_{\text {dry. }}$. Extension of vorticity variation region toward the lower wall at the nozzle corner causes the position of $\delta_{\text {dry }}$ to move away from the upper wall as the measurement position approaches the sidewall. Therefore, it can be said that the change in density has a strong correlation with the change in vorticity. Further, $\delta_{\text {dry.max }}$ becomes larger at $x / H=2$ compared with that of $x / H=0$.

Figure 6 shows a schlieren photograph [22] of the rectangular nozzle in case of dry air. In this figure, the end of the white band from the solid wall in the direction perpendicular to the knife-edge indicates the thickness of experimental density gradient $\delta_{\text {dry.exp. }}$. Comparison between the calculated $\delta_{\text {dry.max }}$ and measured $\delta_{\text {dryexp }}$ [22] has been made at four positions $(x / H=0,1,1.4$, and 2$)$. It is found 


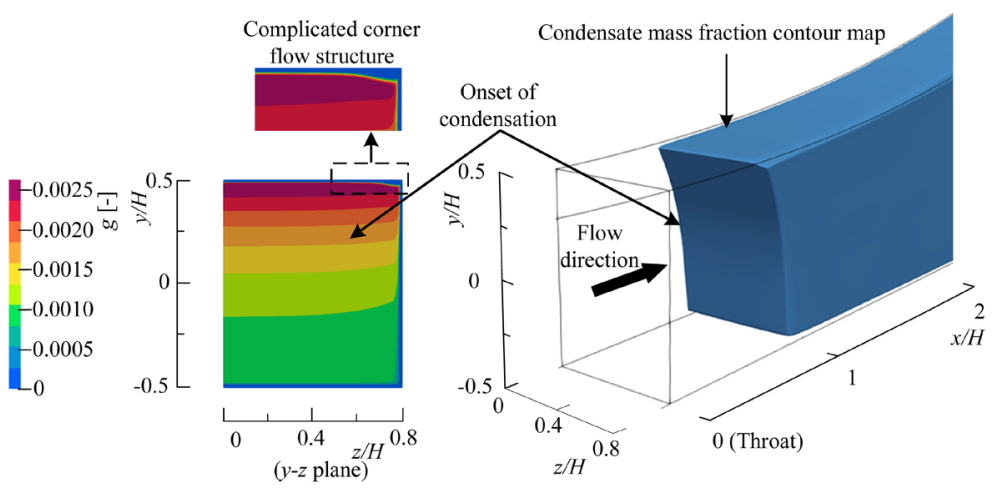

Figure 4. Three-dimensional contour map of condensate mass fraction $g$ with onset of non-equilibrium homogeneous condensation.
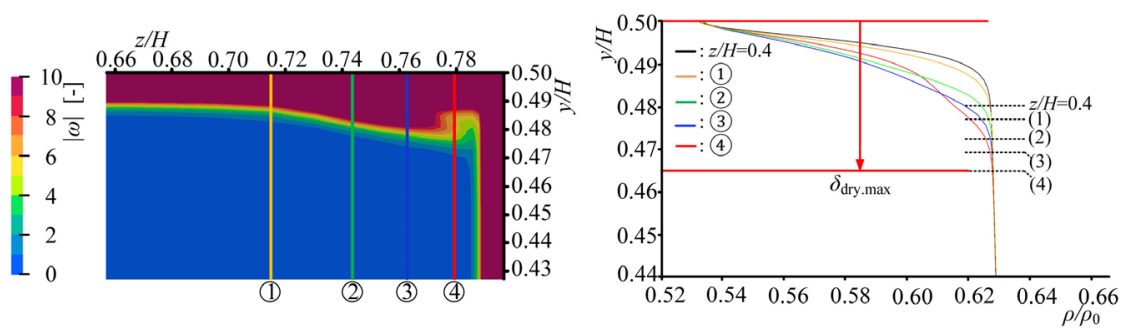

(a)
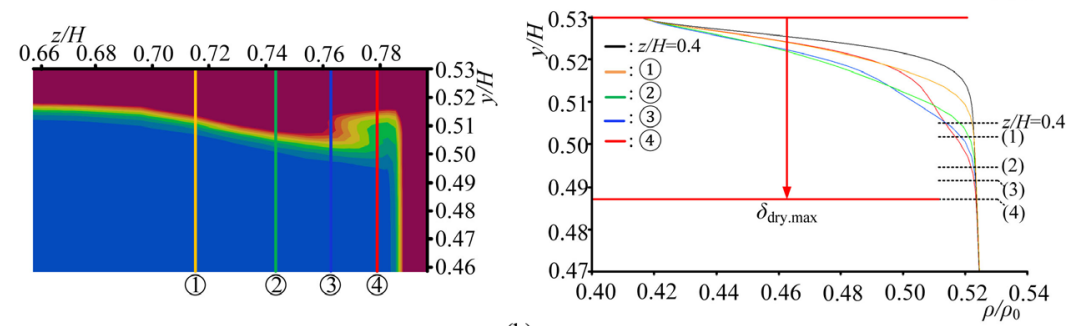

(b)
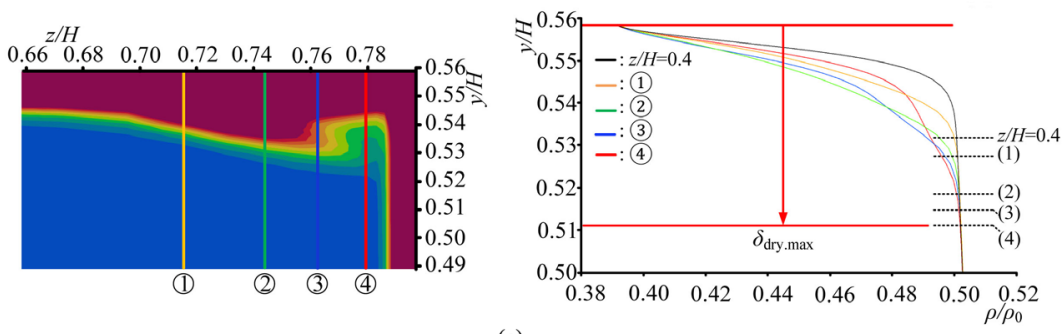

(c)


(d)

Figure 5. Contour maps of vorticity and $\rho / \rho_{0}$ profiles at $x / H=0,1,1.4$, and 2 (dry air). (a) $x / H=0$; (b) $x / H=1$; (c) $x / H=1.4$; (d) $x / H=2$. 
that the value of $\delta_{\text {dry.max }}$ is almost the same as the $\delta_{\text {dry.exp }}$ of schlieren photograph at each position. These results confirm the relation between the experimental datum and present simulation outputs.

Four cross-sectional planes $(x / H=0,1,1.4$, and 2$)$ in Figure 7 have been used to show the correlation between the contour maps of vorticity $(\omega)$ and density distributions in case of moist air. At each cross-sectional plane, density distributions in the $y$-axis direction are shown at the same positions as of Figure 5 near the corner of the nozzle upper wall. It can be seen that condensation causes an increase in flow density when the flow moves toward the nozzle corner. The irregularity in the density profile could have been induced by the onset of non-equilibrium homogeneous condensation at the nozzle corner flow. From these figures, it can be deduced that the location of the $\delta_{\text {moist }}$ i.e., minimum absolute value of density gradient $(d \rho / d y)$ departs further from the upper wall as the measuring position reaches the sidewall. This is attributed to the extension of vorticity variation region toward the lower wall at the corner of the nozzle. Further, $\delta_{\text {moistmax }}$ which indicates the maximum value of $\delta_{\text {moist }}$ becomes larger at $x / H$ $=2$ compared with that of $x / H=0$.

In Table 1, the thickness of $\delta_{\text {drymax }}$ and $\delta_{\text {moistmax }}$ in cases of dry air and moist air are indicated at $x / H=0,1,1.4$ and 2. From this table, it can be seen that the non-equilibrium homogeneous condensation that occurred downstream of the nozzle throat reduces the thickness compared with the $\delta_{\text {drymax }}$ at $x / H=1$ and 1.4. Therefore, it can be deduced that the latent heat released by the non-equilibrium homogeneous condensation suppresses the development of longitudinal vortices near the nozzle corner.

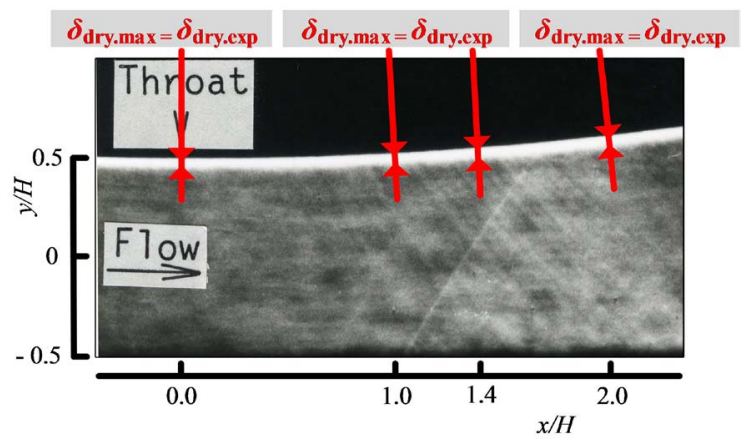

Figure 6. Schlieren photograph of $\rho / \rho_{0}$ in case of dry air (knife edge position:

Table 1. Thickness of $\delta_{\text {dry.max }}$ and $\delta_{\text {moistmax }}$ in cases of dry air and moist air.

\begin{tabular}{cccc}
\hline $\begin{array}{c}\text { Measurement } \\
\text { positions }\end{array}$ & $\begin{array}{c}\text { Thickness for dry air } \\
\delta_{\text {dry.max }}[\mathrm{mm}]\end{array}$ & $\begin{array}{c}\text { Thickness for moist air } \\
\delta_{\text {most.max }}[\mathrm{mm}]\end{array}$ & $\begin{array}{c}\delta_{\text {dry.max }}-\delta_{\text {moss.max }} \\
\delta_{\text {dry.max }}\end{array}$ \\
\hline$X / H=0$ & 0.8568 & 0.8568 & 0 \\
$X / H=1$ & 0.9996 & 0.952 & 4.76 \\
$X / H=1.4$ & 1.1186 & 0.9044 & 19.15 \\
$X / H=2$ & 1.19 & 1.0472 & 12.0 \\
\hline
\end{tabular}



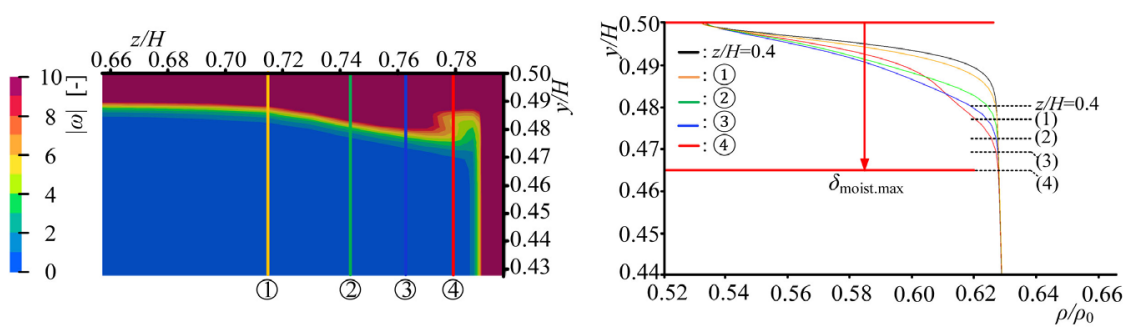

(a)
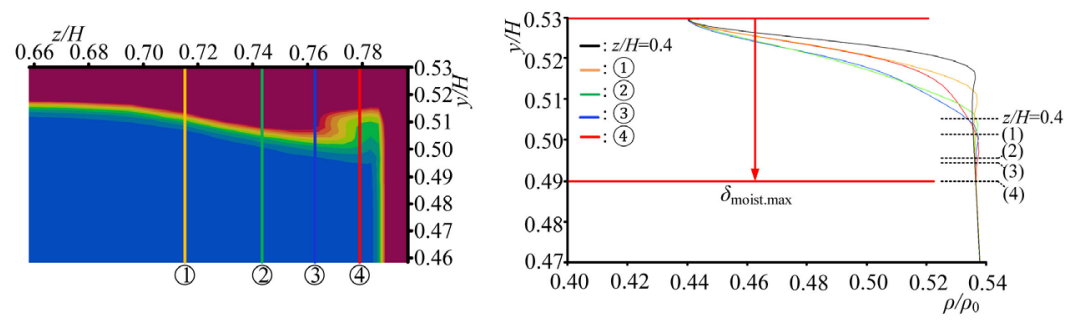

(b)
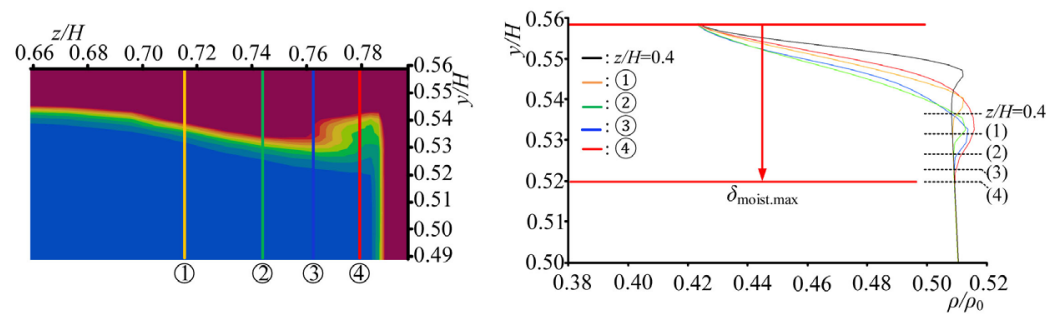

(c)
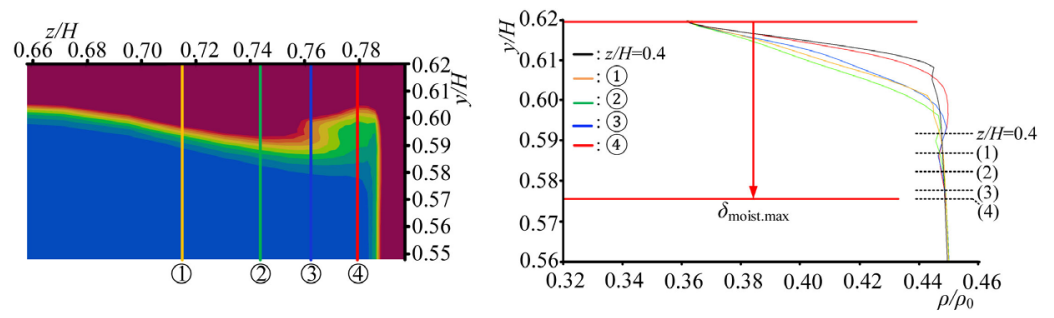

(d)

Figure 7. Contour maps of vorticity and distributions of $\rho / \rho_{0}$ at $x / H=0,1,1.4$, and 2 (moist air). (a) $x / H=0$; (b) $x / H=1$; (c) $x / H=1.4$; (d) $x / H=2$.

\section{Conclusions}

This numerical study has been set out to investigate the effect of non-equilibrium homogeneous condensation on the corner flow of a supersonic rectangular arc nozzle. The results obtained are as follows:

1) An increase of static pressure downstream of the nozzle throat in case of moist air indicated the non-equilibrium homogeneous condensation. Simulated results of static pressure distribution were in good agreement with the experimental results.

2) Change in density at the nozzle corner flow had a strong correlation with the change in vorticity. This is because the extension of vorticity variation region toward the lower wall caused the position of density gradient with minimum 
absolute value to move further away from the upper wall.

3) In the range of the nozzle corner, surface of condensation onset had a complicated structure due to an interaction between the boundary layer and longitudinal vortex.

4) Latent heat released by the non-equilibrium homogeneous condensation suppressed the development of longitudinal vortices near the nozzle corner which resulted in the reduced thickness of $\delta_{\text {moist.max }}$.

\section{Conflicts of Interest}

The authors declare no conflicts of interest regarding the publication of this paper.

\section{References}

[1] Görtler, H. (1954) On the Three-Dimensional Instability of Laminar Boundary Layers on Concave Walls. NACA TM 1375; a Translation of Göttingen, G.D.W. and Math, A.D.N. (1940) Über eine dreidimensionale Instabilität laminarer Grenzschichten an konkaven Wänden. Bd. 2, Nr. 1.

[2] Wang, W.P., Lin, R.S. and Malik, M.R. (1997) Control of Corner Flow Vortices by Geometry Shaping in Mach 2.4 Rectangular Nozzles. AIAA, 97-2228, 145-155. https://doi.org/10.2514/6.1997-2228

[3] Sharma, O.P. and Butler, T.L. (1987) Predictions of Endwall Losses and Secondary Flows in Axial Flow Turbines Cascades. ASME Journal of Turbomachinery, 109, 229-236. https://doi.org/10.1115/1.3262089

[4] Hawthorne, W.R. (1951) Secondary Circulation of Fluid Flow. Proceedings of Royal Society A, 206, 374-387.

[5] Wegener, P.P. and Mach, L.M. (1958) Condensation in Supersonic and Hypersonic Wind Tunnels. Advances in Applied Mechanics, 5, 307-447. https://doi.org/10.1016/S0065-2156(08)70022-X

[6] Heybey, W. (1947) Analytical Treatment of Normal Condensation Shock. NACA Technical Memorandum; A Translation of "Analytische Behandlung des geraden Kondensationsstosses," Heeres-Versuchsstells, Peenemunde, Archiv Nr. 66/72, Marz 30,1942 .

[7] Abraham, F.F. (1974) Homogeneous Nucleation Theory. Academic Press, New York and London. https://doi.org/10.1063/1.3129038

[8] Feder, J., Russell, K.C., Lothe, J. and Pound, G.M. (1966) Homogeneous Nucleation and Growth of Droplets in Vapors. Advances in Physics, 15, 111-178.

https://doi.org/10.1080/00018736600101264

[9] Becker, J.V. (1950) Results of Recent Hypersonic and Un-Steady Flow Research at the Langley Aeronautical Laboratory. Journal of Applied Physics, 21, 619-628. https://doi.org/10.1063/1.1699720

[10] Moore, M.J., Walters, P.T., Crane, R.I. and Davidson, B.J. (1973) Predicting the Fog-Drop Size in Wet-Steam Turbines. Proceedings of the IMechE Conference on Heat and Fluid Flow in Steam and Gas Turbine Plant, Coventry, April 1973, C37/73, 101-109.

[11] Perrell, E.R., Erickson, W.D. and Candler, G.V. (1996) Numerical Simulation of Nonequilibrium Condensation in a Hypersonic Wind Tunnel. AIAA Journal of Thermo-Physics and Heat Transfer, 10, 277-283. https://doi.org/10.2514/3.784 
[12] Pouring, A.A. (1965) Thermal Choking and Condensation in Nozzles. Physics of Fluids, 8, 1802-1810. https://doi.org/10.1063/1.1761112

[13] Wegener, P.P. and Pouring, A.A. (1964) Experiments on Condensation of Water Vapor by Homogeneous Nucleation in Nozzles. Physics of Fluids, 7, 352-361. https://doi.org/10.1063/1.1711206

[14] Daum, F.L. (1963) Air Condensation in a Hypersonic Wind Tunnel. AIAA Journal, 1, 1043-1046. https://doi.org/10.2514/3.1722

[15] Roe, P.L. (1981) Approximate Riemann Solvers, Parameter Vectors, and Difference Schemes. Journal of Computational Physics, 43, 357-372.

https://doi.org/10.1016/0021-9991(81)90128-5

[16] Chakravarthy, S.P. (1984) Relaxation Methods for Unfactored Implicity Upwind Schemes. AIAA Paper, 84-0165. https://doi.org/10.2514/6.1984-165

[17] Michael, H. (1999) Instationäre Phänomene in homogen/heterogen kondensierenden Duüsen und Turbinenströmungen. Dissertation, Fakultät für Maschinenbau, Universität Karlsruhe (TH), Karlsruhe.

[18] Adam, S. (1999) Numerische und Experimentelle Untersuchung Instationärer Düsenströmungen mit Energiezufuhr durch Homogene Kondensation. Dissertation, Fakultät für Maschinenbau, Universität Karlsruhe (TH), Karlsruhe.

[19] Frenkel, J. (1946) Kinetic Theory of Liquids. Oxford University Press, Oxford.

[20] Keith, W. and Davor, C. (2008) A Three-Equation Eddy-Viscosity Model for Reynolds-Average Navier-Stokes Simulations of Transitional Flow. Journal of Fluids Engineering, 130, 145-155. https://doi.org/10.1115/1.2979230

[21] Coupland, J. (1990) Ercoftac Special Interest Group on Laminar to Turbulent Transition and Retransition: T3a Test Cases. Technical Report, ERCOFTAC.

[22] Matsuo, S. (1988) Study on the Effect of Condensation on Supersonic Internal Flow. Ph.D. Thesis, Graduate School of Science and Engineering, Kyushu University, Fukuoka. (In Japanese) 


\section{Nomenclature}

\begin{tabular}{ll}
$p$ & Static pressure \\
$Q$ & Conservative vector \\
$E, F, G$ & Inviscid flux vector \\
$E_{\mathrm{v}}, F_{\mathrm{v}}, G_{\mathrm{v}}$ & Viscosity flux vector \\
$I$ & Turbulence vector \\
$S$ & Condensation vector \\
$T$ & Temperature \\
$R$ & Radius of curvature \\
$\rho$ & Density \\
$u$ & Velocity component in $x$-axis direction \\
$V$ & Velocity component in $y$-axis direction \\
$W$ & Velocity component in $z$-axis direction \\
$n$ & Number of condensate particles \\
$g$ & Condensate mass fraction \\
$k_{\mathrm{t}}$ & Turbulent kinetic energy \\
$k_{\mathrm{l}}$ & Laminar kinetic energy \\
$\omega$ & Specific dissipation rate \\
$\phi$ & Relative humidity \\
$H$ & Nozzle throat height \\
$\sigma$ & Surface tension \\
$I_{\mathrm{F}}$ & Critical cluster radius \\
\hline $\mathrm{m}$ & Nubleation rate \\
0 & Droplet average radius \\
\hline & Stagnation point \\
\hline & Mixture of air and vapor \\
\hline & Liquid \\
\hline &
\end{tabular}

